\title{
TEM and Atom Probe Microscopy in the Development of Magnetic Multilayer Nano-Structures
}

\author{
P. M. Rice*, T. Lin**, R. E. Fontana Jr.**, E. A. Delenia* \\ *IBM Almaden Research Center, 650 Harry Road, San Jose CA, 95120 \\ **Hitachi Global Storage Technology, 650 Harry Road, San Jose, CA 95120
}

While for some "nano technology" may be just a buzz word promising great things in the future, for the Hard Disk Drive Industry its been a reality for some time. The sensors that read the ones and zeros stored on your computers hard disk now have dimensions at or below the $100 \mathrm{~nm}$ regime. Development of such small structures has become heavily dependent on FIB/TEM to answer processing and materials development questions.

Figure 1 shows a TEM micrograph of a Magnetic Tunnel Junction (MTJ) device which has a stack height of about $50 \mathrm{~nm}$ and a sensor width of about $200 \mathrm{~nm}$. The height of the sensor stack sets a minimum spacing between the magnetic shields ( also known as the 'gap'), which in turn limits the spacing of the bits to be read since the sensor must react to only one bit transition at a time. The smaller the gap, the closer the bits can be written together increasing storage density. The width of the sensor effects the spacing between lines of data on the disk. Thus the smaller the width of the sensor, the closer together the lines of data can be written, again increasing storage density. The hardbias is used to help magnetically stabilize the 'free' layer.

The introduction of the Focussed Ion Beam (FIB) instrument has allowed us to do site specific TEM analysis from any one of the approximately 20,000 devices on a wafer, and at any step in the process of the devices development. TEM (and more recently atom probe microscopy) has been used to study a vast number of device properties such as interface roughness. Very small differences in the smoothness of certain layers significantly effects the magnetic properties of these multilayer structures. Figure 2 includes TEM micrographs which show that the deposition technique used to deposit the layers can have small but significant effects on the resultant interface roughness. The layers shown in Figure 2a) where deposited using an Ion Beam Deposition (IBD) technique, while those of Figure 2b) where deposited using Physical Vapor Deposition (PVD) techniques. The interface between the insulating tunnel barrier material (Al-O) and the CoFe pinned layer just below it shows that the IBD method created a much smoother interface than the PVD method. The magnetic properties for the IBD specimen were also superior.

The problem with TEM, which we hope atom probe microscopy can help overcome, is that any image is a through-specimen projection. Most the devices sold today are not the MTJ type sensor shown here, but are of the Giant Magnetoresistance (GMR) type device. The magnetic stack is very similar to the one presented in Figure 2 except that the Al-O layer is replaced with $\mathrm{Cu}$. In that case there is no contrast mechanism to show the small differences in interface roughness that make such a difference to the properties, and thus usefulness of the device. To help study these effects further, IBM has initiated an atom probe project so that we may be more involved with the rapidly improving world of atom probe microscopy. 


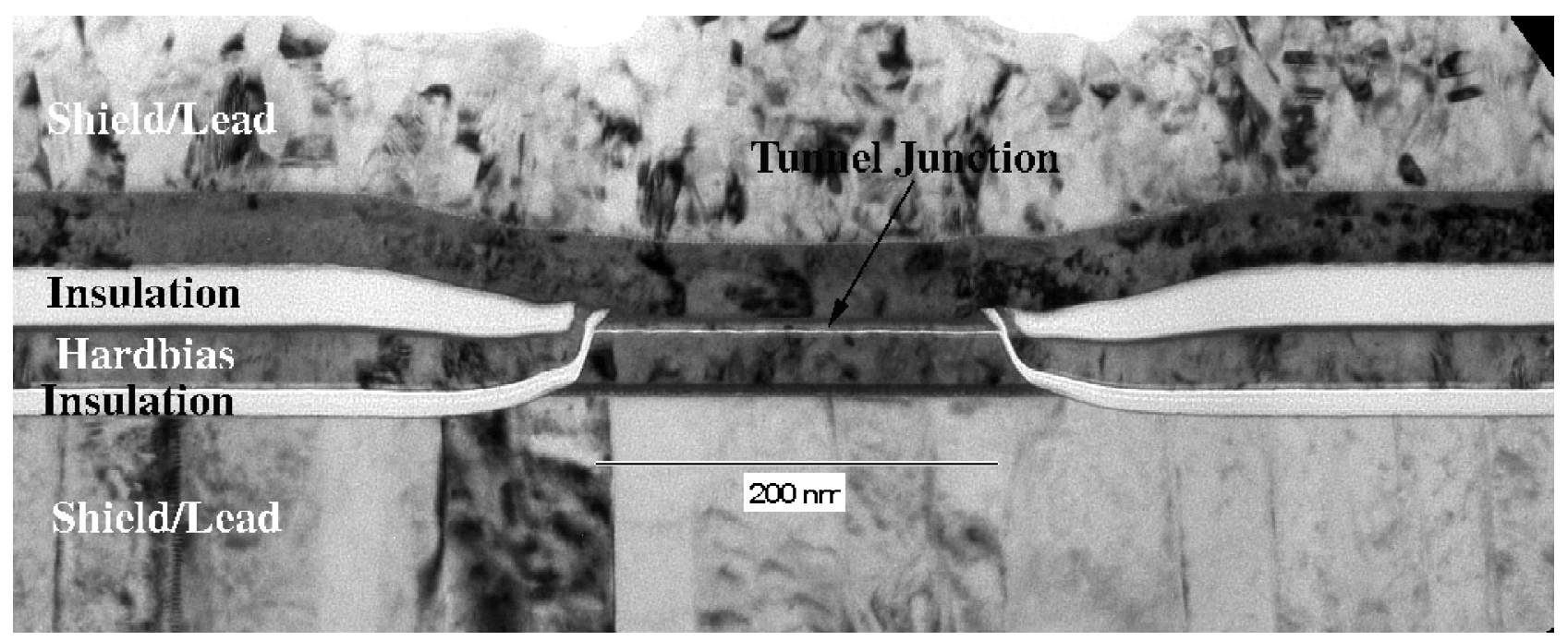

Fig. 1. A TEM micrograph of a $200 \mathrm{~nm}$ magnetic tunnel junction read sensor for a computer hard drive.
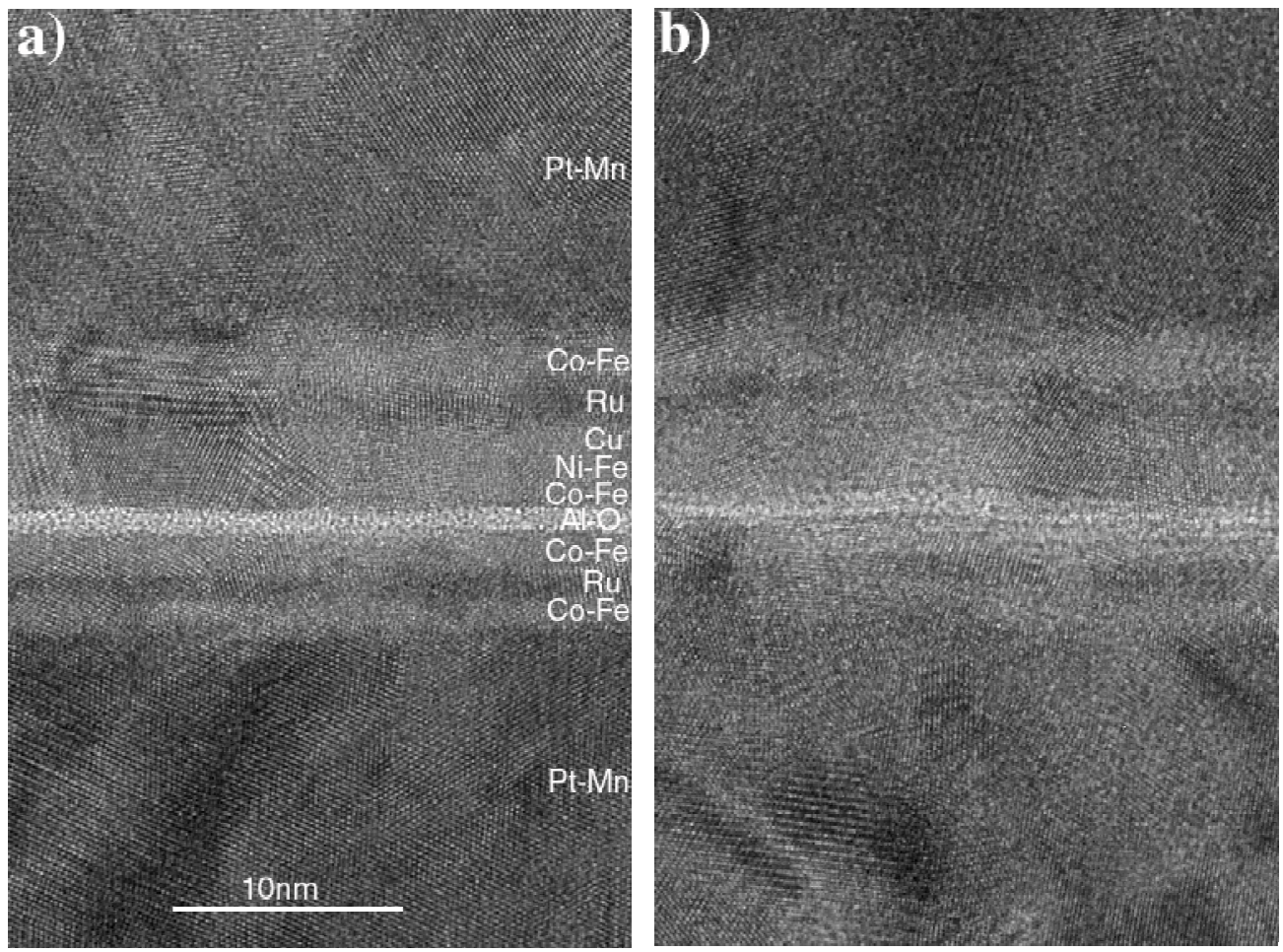

\begin{tabular}{|c|} 
c) \\
bias \\
antiferromagnetic \\
layer \\
bias layer \\
decoupling layer \\
free layer \\
tunnel barrier \\
second pinned layer \\
spacer layer \\
first pinned layer \\
antiferromagnetic \\
layer
\end{tabular}

Fig. 2. a) and b) are TEM micrographs of magnetic tunnel junction stacks which were deposited using IBD and PVD deposition techniques respectively. The IBD technique resulted in much smoother interface. c) gives a brief description of each layers function. 